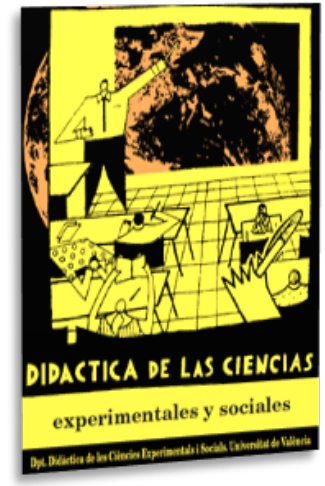

\title{
Agenda 2030 y representación socioemocional del medio ambiente en la Educación Primaria
}

\author{
The 2030 Agenda and socio-emotional \\ representation of the environment in Primary \\ Education
}

DOI: $10.7203 / D C E S .41 .19413$

\author{
Laura Sánchez Giménez \\ Universitat de València, lausanchezgimenez93@gmail.com \\ ORCID iD: https://orcid.org/0000-0002-6712-3326 \\ Antonio José Morales Hernández \\ Universitat de València, Antonio.J.Morales@uv.es \\ ORCID iD: https://orcid.org/0000-0002-7736-0827 \\ Diana Santana Martín \\ Universitat de València, Diana.Santana@uv.es
}

\begin{abstract}
RESUMEN: ¿Existe una correlación entre las emociones y representaciones sociales que los escolares tienen sobre el medio ambiente capaz de incidir en el desarrollo de los Objetivos de Desarrollo Sostenible de la Agenda 2030? A partir de esta inquietud hemos planteado un estudio de casos en tercer ciclo de Educación Primaria. Para ello hemos solicitado a 117 estudiantes que realicen un dibujo sobre el medio ambiente asociándolo a la emoción que les provoca. Los resultados evidencian tendencias de correlación entre las emociones básicas y las representaciones sociales del medio ambiente. Se distingue la asociación de representaciones naturalistas y antropocéntricas del medio ambiente con emociones positivas o neutras. Así mismo, las representaciones globalizantes se identifican con una percepción de la problemática ambiental desde una doble óptica emocional: más angustiada o más serena. Todo ello muestra indicios de la existencia de una serie de etapas en la interiorización de la Educación Ambiental.
\end{abstract}

Palabras Clave: representación mental, emoción, enseñanza primaria, educación ambiental, educación de la percepción

ABSTRACT: Is there a correlation between the emotions and social representations that schoolchildren have about the environment capable of influencing the implementation of the Sustainable Development Goals of the 2030 Agenda? Based on this concern, we have proposed a case study in the third cycle of Primary Education. To do this, we have asked 117 students to draw a picture of the environment, associating it with the emotion that provokes in them. The results show correlation trends between basic emotions and social representations of the environment. The association of naturalistic and anthropocentric representations of the environment with positive or neutral emotions is distinguished. Likewise, globalizing representations are identified with the perception of environmental problems from a double perspective: a more anguished or a more serene emotional experience. All this shows signs of the existence of a series of stages in the internalization of Environmental Education.

KEYWORDS: mental representation, emotion, primary education, environmental education, perception education

Fecha de recepción: enero de 2021

Fecha de aceptación: abril de 2021

Este artículo se inscribe dentro del proyecto: "Las representaciones sociales de los contenidos escolares en el desarrollo de las competencias docentes", Proyectos I+D de Generación de Conocimiento y Fortalecimiento Científico y Tecnológico del Sistema I+D+I (Ministerio de Ciencia, Innovación y Universidades), con referencia PGC2018-094491-B-C32, cofinanciado con fondos FEDER de la UE. 


\section{INTRODUCCIÓN}

La Agenda 2030, a través de 17 Objetivos de Desarrollo Sostenible (ODS) y 169 metas, plantea la necesidad de transformar el mundo. El ODS 4, referente a la educación inclusiva y de calidad, destaca entre otras finalidades:

De aquí a 2030, asegurar que todos los alumnos adquieran los conocimientos teóricos y prácticos necesarios para promover el desarrollo sostenible, entre otras cosas mediante la educación para el desarrollo sostenible y los estilos de vida sostenibles, los derechos humanos, la igualdad de género, la promoción de una cultura de paz y no violencia, la ciudadanía mundial y la valoración de la diversidad cultural y la contribución de la cultura al desarrollo sostenible. (Naciones Unidas, 2015, p. 20)

Además, estamos de acuerdo con Bautista-Cerro, Murga-Menoyo y Novo (2019) en que la Educación Ambiental está en proceso de construcción y que la sostenibilidad es un recurso fundamental para fomentar este proceso desde una acción educativa y ciudadana eficaz (Gil-Pérez y Vilches, 2019). En este sentido, se puede decir que la Educación Ambiental favorece la ciudadanía crítica (Santana, Morales y Souto, 2019). Pero, para abordar esta transformación desde los centros educativos es preciso, en primer lugar, analizar la representación social que el alumnado tiene del mismo, valorando las emociones que siente y que le pueden motivar para pasar a la acción y mejorar el Medio Ambiente desde una perspectiva ecológica y social. En este sentido, es de vital importancia la aportación de las escuelas a este cometido, al incluir en el curriculum contenidos que permitan una educación ecosocial transformadora (González, coord., 2018). En este contexto, recuperamos un trabajo de investigación inédito (Sánchez, 2016) enmarcado dentro del Máster Universitario en Investigación en Didácticas Específicas de la Universitat de València, por su posible incidencia en la sostenibilidad curricular al vincular las emociones y la educación ambiental.

Según la Declaración de Tbilisi "la educación ambiental debería suscitar una vinculación más estrecha entre los procesos educativos y la realidad" (Unesco, 1977, p. 29). Tonucci (1993) alude a la necesidad de que esta propuesta educativa, en el ámbito de la Educación Ambiental, sea coherente. Para ello es preciso conjugar las competencias para la vida desde la Educación Emocional (Bisquerra, 2003) con la inteligencia ecológica (Goleman, 2009) desde la Educación Ambiental. Ciertos autores como Soler y Conangla (2013), acuñan esta relación a través del término "ecología emocional". Si bien, es preciso concretar qué entendemos por Medio Ambiente a partir de nuestras representaciones sociales (en adelante RRSS), así como identificar las emociones que pueden llegar a generar conductas sostenibles hacia el Medio Ambiente. Esta cuestión, aplicada al ámbito de la educación formal, lleva a formularnos la siguiente pregunta de investigación: ¿existe una correlación entre las emociones y las RRSS que los escolares tienen sobre el Medio Ambiente capaz de incidir en la implementación de la Agenda 2030?

Con este propósito y visto que la finalidad del trabajo es comprobar la interdependencia entre la Educación Ambiental y la Emocional por su presumible incidencia en el desarrollo de los ODS de la Agenda 2030, la hipótesis planteada pretende verificar la posible relación entre las RRSS del concepto de Medio Ambiente y las emociones generadas en el alumnado a partir de un estudio de casos en tercer ciclo de Educación Primaria por su repercusión en la implementación de la Agenda 2030 en el ámbito escolar. Para ello hemos solicitado a una muestra compuesta por 117 alumnos/as del tercer ciclo de Educación Primaria ( $5^{\circ}$ y $6^{\circ}$ curso) la elaboración de un dibujo sobre lo que entienden por Medio Ambiente, señalando la emoción que les provoca. Todo ello con la intención de mostrar las posibles tendencias de correlación entre las emociones básicas y las RRSS del Medio Ambiente propuestas por la clasificación de Reigota (1990). Los objetivos de esta investigación son: 
1- Analizar las RRSS del concepto del Medio Ambiente del alumnado de tercer ciclo de Educación Primaria y su clasificación según Reigota (1990).

2- Distinguir la vinculación existente entre las distintas RRSS del Medio Ambiente y su correlación con un determinado tipo de emociones (Ekman y Oster, 1981).

3- Evidenciar la presumible incidencia de la Educación Emocional en el aprendizaje de la Educación Ambiental y su incidencia escolar.

4- Valorar como puede afectar todo ello al posible desarrollo de la Agenda 2030 en el ámbito escolar.

\section{FUNDAMENTACIÓN TEÓRICA: REPRESENTACIONES SOCIALES Y EDUCACIÓN EMOCIONAL}

En el ámbito de nuestra línea de investigación sobre "Educación Socioambiental y Didáctica del Patrimonio: de lo local a lo global" (Socials UV) ${ }^{1}$ hemos podido constatar la necesidad planteada por la Agenda 2030 para abordar la problemática socioambiental de manera holística, con la finalidad de movilizar conductas pro-activas transformadoras para mejorar el mundo. Lo que se traduce, a su vez, en la conveniencia de educar la percepción de las personas desde el enfoque globalizado de la realidad para favorecer la comprensión de la complejidad del entorno (local y global) mediante la interrelación de la dimensión natural y social. Ciertos autores manifiestan que no es posible alcanzar los ODS de la Agenda 2030 si se obvia la vinculación que existe entre todos ellos aludiendo a la ciencia de la sostenibilidad, por su carácter orientador, para fomentar la transversalidad entre las distintas disciplinas (Vilches y Gil, 2021). Estamos de acuerdo con ello, pero desde el ámbito social creemos que es necesario repensar las RRSS y su vinculación con las emociones relacionadas con el Medio Ambiente para estudiar este concepto desde el espacio vivido. En este sentido, nos preocupa que la dimensión medioambiental sea un producto más de la sociedad de consumo ofertado como "lo verde" a través de campañas de marketing. Nos inquieta que se transmita como percibir, pensar y sentir el Medio Ambiente, sin pararse a reflexionar como construyen las personas este concepto.

\subsection{Representaciones sociales para transformar el mundo}

Este trabajo se aborda desde la teoría de las RRSS, desarrollada principalmente por Moscovici (1961), la cual ha sido reconocida en la psicología social y en el resto de Ciencias Sociales por su legítimo carácter científico (Villarroel, 2007). La definición de RRSS de Moscovici se diferencia de las representaciones colectivas de Durkheim (1898) para atribuirle un carácter individual y heterogéneo que incluye la diversidad de los miembros de un mismo grupo social y la pluralidad de sus construcciones simbólicas. De este modo, se manifiesta no solo su carácter social sino también su componente individual. Así mismo, las aportaciones de Jodelet (1986) le atribuyen el carácter psicológico como pensamiento socialmente desarrollado para contribuir a la construcción de una realidad común.

Por consiguiente, conocer los elementos constitutivos de las RRSS sobre el concepto de Medio Ambiente y su relación con el desarrollo de emociones es relevante, en tanto que permite indagar en las concepciones que influyen en el comportamiento por su repercusión en los procesos de interacción con el medio. Esto nos daría las claves para poder establecer líneas de trabajo encaminadas a favorecer una educación para la transformación ecosocial (González, coord., 2018). Desde esta perspectiva, una línea de trabajo de Social (S) relaciona las representaciones sociales con la geografía de la percepción (García, Morote y Souto, 2019; Souto y García, 2019).

\footnotetext{
${ }^{1}$ Ver http://socialsuv.org/investigacion/
} 
En este contexto, los procesos de comunicación y pensamiento social son claves para desarrollar un tipo de RRSS determinado que influye en la manera en la que los escolares, como parte de la ciudadanía, actúan. Por lo tanto, éstas se entienden como estructuras específicas del conocimiento del sentido común. El carácter práctico de las RRSS asigna especial relevancia a la comprensión y explicación de los hechos de la vida cotidiana. Esto favorece la intervención, no solo en la construcción social de la realidad (Villarroel, 2007), sino también en su transformación, puesto que integra el análisis de los procesos de participación social del sujeto (Jodelet, 1994) para relacionarse con la "acción social" (Carretero, 2010).

Por tanto, las RRSS son una modalidad particular de conocimiento, cuya función es el desarrollo de comportamientos y la comunicación entre los individuos. Rigen las relaciones que establece el ser humano con su medio natural y social, estableciéndose como guías para la acción (Abric, 2001) que facilitan el entendimiento y la explicación de una realidad definida por un determinado marco social de referencia. De esta manera, las RRSS se conforman como un corpus organizado que permite hacer inteligible la realidad física y social del individuo que lo integra en un grupo mediante la generación de intercambios (Moscovici, 1979). Así mismo, las RRSS se asocian directamente a la actitud. De este modo, las actitudes manifiestan la respuesta emocional hacia el objeto o hecho social. Son el elemento más primitivo de las RRSS (Araya, 2002) y se complementan con un comportamiento o reacción afectiva. Por tanto, se incluye la actitud que el individuo adopta con respecto a un objeto determinado y la respuesta emocional que desencadena un comportamiento concreto hacia dicho objeto. Por lo tanto, en la conformación de las RRSS intervienen dos tipos de emociones: las relacionadas con el componente afectivo de las actitudes favorables que guían las prácticas cotidianas y las relacionadas con el componente afectivo desfavorable que se orientan hacia la denuncia (Calixto, 2010) y, por tanto, que favorecen el cambio social.

Como consecuencia, cambiar una representación social pasa necesariamente por la deconstrucción de los discursos mediante su lectura crítica que permita discutir, dialogar y confrontar con otras interpretaciones de la realidad y situaciones cotidianas (Reigota, 2010; Souto y García, 2017). En coherencia, Reigota (2010) apunta que desde la Educación Ambiental se debe promover que las RRSS se expresen, se escuchen y se debatan, por su relevancia en el desarrollo de actitudes que favorecen el ejercicio de la participación democrática y la intervención ciudadana.

Por otra parte, las investigaciones reconocen que las emociones experimentadas por el sujeto cuando entra en contacto con el objeto, en este caso el Medio Ambiente, intervienen en su construcción cognitiva pero también influyen en su comportamiento, tanto social como moral. Por tanto, a través de las RRSS que se construyen en torno a un objeto, las personas dotan de sentido las prácticas cotidianas (Calixto, 2010). De esta manera, el entorno se debe interpretar teniendo en cuenta que nunca habrá una única lectura y que ésta estará vinculada a las prácticas sociales y experiencias de cada comunidad (Morales et al., 2014). Tal y como apunta Souto (2011):

Si una persona es capaz de reflexionar con argumentos conceptuales sobre esta manera de conducir las emociones y los afectos, será tanto más competente para participar en la tarea ciudadana de ordenar el territorio donde vive. En esencia, esta reflexión argumental es el arma cargada de futuro de la geografía escolar: colaborar en un proyecto de ciudadanía crítica y responsable con las acciones humanas sobre el planeta Tierra. (Souto, 2011, p. 56)

A modo de recapitulación nos preocupa la manera de incidir de la Educación Ambiental en el desarrollo de la ciudadanía crítica (Santana, Morales y Souto, 2019) para que las agendas institucionales puedan ser vivenciadas a través de las agendas personales. Por ello hemos analizado la evolución de los conceptos de sostenibilidad y solidaridad (Parra, Morales y Caurín, 2020) mostrando la necesidad de reflexionar sobre las diversas RRSS de ambos conceptos y la vinculación existente entre los mismos. Todo ello con la finalidad de apreciar la posible alineación de estas percepciones con el enfoque holístico que propone la Agenda 2030 para transformar el mundo. 


\subsection{La relevancia de las emociones en la Educación Ambiental}

Scott (2010) propone un modelo de aprendizaje que aboga por un mayor énfasis pedagógico en el dominio afectivo, en la función de las emociones y de lo intuitivo. Dicho modelo no se centra únicamente en el desarrollo de habilidades cognitivas, sino sociales, incluyendo el aprendizaje emocional. Por tanto, se presta especial atención a la dimensión emocional, por su relevancia con la sensibilidad ambiental. De este modo, Scott (2010) evidencia que las personas no transforman su comportamiento únicamente al recibir nueva información, por lo que no basta con la adquisición de conocimiento teórico. Intervienen otro tipo de variables que son las que generan la representación cotidiana que generamos del mundo. En relación directa con las RRSS desarrolladas, el autor evidencia la relevancia que éstas asumen para cambiar los comportamientos hacia modelos más proambientales.

En esta línea, ya en 1999, Nundy demostró la relación existente entre los dominios afectivo y cognitivo existentes que experimentaban estudiantes de Educación Primaria mediante las visitas al medio natural en el sur de Inglaterra. De este modo, se evidencia la existencia de vínculos que conectan las experiencias, con los conocimientos y valores que brindan oportunidades para que los escolares participen en actividades ambientales. De hecho, el tratamiento de las emociones se está considerando cada vez más como objeto de estudio de las investigaciones educativas. Algunas de ellas ponen de manifiesto las relaciones existentes entre la naturaleza y el bienestar emocional que centran el foco de atención en los efectos beneficiosos y reparadores que aporta el contacto con el medio natural para el ser humano. Siguiendo esta línea, incluso se cuenta con investigaciones como la desarrollada por Seresinhe, Preis y Moat (2015) que cuantifican las características estéticas del paisaje para demostrar que los espacios más verdes ejercen una mayor influencia positiva en el bienestar y salud de las personas. Así mismo, existen investigaciones que relacionan las conductas proambientales al bienestar emocional (Amérigo, García y Sánchez, 2013) que ponen de manifiesto que cuando las personas incorporan el concepto de sí mismos al de naturaleza, manifiestan un mayor bienestar emocional. Con lo cual, se evidencia la necesidad de relacionar el bienestar individual con el medioambiental.

El estudio elaborado por Hartig, Kaiser y Bowler (2001) explica que una gran parte de los comportamientos proambientales de las personas se justifican por la percepción que poseen sobre las propias cualidades restauradoras de los medios naturales, adquiriéndose a modo de favor devuelto. En este sentido, se resalta la especial relevancia que adquiere la Educación Ambiental por sus consecuencias positivas en la salud del Medio Ambiente y bienestar de las personas. Sin embargo, también se cita la investigación de Lindenberg y Steg (2007) para demostrar cómo este tipo de conductas proambientales pueden minimizarse o dejar de practicarse cuando implican una pérdida de placer personal o esfuerzo adicional. Amérigo, Aragonés y García (2012) relacionan los elevados grados de inclusión en la naturaleza con una alta vinculación emocional e interés por el Medio Ambiente, mientras que las relaciones se invierten en los casos de antropocentrismo y de apatía medioambiental. De cualquier modo, para que se produzca algún cambio positivo para el Medio Ambiente, la ciudadanía tiene que tomar conciencia previamente de que existe un problema que merece una solución mediante la acción.

En este sentido a pesar de que la emoción se entiende como un estado de disposición para la acción (Frijda, 1988; Milton, 2002), merece la pena valorar hasta qué punto las emociones que se generan en una situación determinada inciden en la necesidad percibida de actuar de una determinada manera. Por ello, no es de extrañar que expertos en Educación Emocional como Bisquerra (2006) incluyan en sus propuestas la Educación Ambiental por su repercusión en el bienestar, incluyendo su dimensión social. De este modo, la Educación Emocional queda definida de la siguiente manera: 
La educación emocional es una innovación educativa que se justifica en las necesidades sociales. La finalidad es el desarrollo de competencias emocionales que contribuyan a un mejor bienestar personal y social. Se concibe la educación emocional como un "proceso educativo, continuo y permanente, que pretende potenciar el desarrollo de las competencias emocionales como elemento esencial del desarrollo integral de la persona, con objeto de capacitarle para la vida. Todo ello tiene como finalidad aumentar el bienestar personal y social. (Bisquerra, 2006, p. 15)

Siguiendo en esta línea, se empieza ya a difundir el término ecología emocional (Soler y Conangla, 2014) para abordar un planteamiento que desde la Educación Emocional trabaja aspectos educativos medioambientales con vistas a construir un modelo de persona emocionalmente ecológica. La capacidad para emocionarse y expresarse que ofrece el contacto directo con el medio natural y social genera las condiciones necesarias para que el individuo se encuentre a disposición de preservar el entorno (García, 2013).

\section{METODOLOGÍA: ESTUDIO DE CASOS EN TERCER CICLO DE EDUCACIÓN PRIMARIA}

El presente estudio de casos se plantea como una investigación cualitativa (Stake, 1999), puesto que la intención no es calcular numéricamente el factor que provoca el problema, sino comprender cuál es el motivo que lo induce y así poder actuar con respecto a éste. Según Yin (1981, p. 59) "The different types of case studies that are possible (exploratory, descriptive, and explanatory)". En este trabajo predomina el carácter exploratorio respecto a la relación existente entre las emociones que los escolares manifiestan sobre el Medio Ambiente y sus RRSS sobre el mismo. Para ello, se ha utilizado como herramienta de investigación el análisis de las diferentes representaciones pictóricas que sobre la Educación Ambiental tiene el alumnado de Educación Primaria, objeto de la muestra de esta investigación, y su posible correlación con las emociones que les suscita. Las representaciones pictóricas han sido utilizadas con anterioridad (Barraza, 1999) en el campo de la investigación educativa para analizar el carácter medioambiental de los dibujos realizados por el alumnado de distintas etapas educativas. Puig, Geli y Besalú (2018) han utilizado recientemente este método para analizar la percepción y proyección de los problemas sociales y ambientales.

Cabe señalar que la metodología utilizada ha sido el estudio de casos, donde la elección de la muestra es criterial al seleccionar escuelas transformadoras de la provincia de Valencia con proyectos innovadores. La justificación de la muestra seleccionada viene definida por el trabajo conjunto que ha realizado el alumnado de estos dos niveles de tercer ciclo a través de proyectos escolares de Educación Ambiental. La contextualización y evolución de alguno de estos proyectos educativos puede consultarse en Autores (Santana, Morales y Henarejos, 2021). La muestra se compone de: 3 grupos de $5^{\circ}$ de Educación Primaria (68 alumnos/as) y 3 grupos de $6^{\circ}$ de Educación Primaria (49 alumnos/as), en total 117 participantes. Todo ello con una finalidad instrumental (Stake, 1999) que nos permita encontrar indicios de la posible vinculación entre la Educación Emocional y la Educación Ambiental, infiriendo con ello mayor protagonismo al fenómeno relacional que pretendemos investigar sobre el propio caso estudiado. Al alumnado se le ha solicitado su colaboración voluntaria en esta investigación, contando en todo momento con la ayuda inestimable del profesorado de los centros participantes.

Por lo que respecta el diseño de la experiencia se ha utilizado una plantilla (Morales, Santana y Sánchez, 2017), para la obtención de las representaciones pictóricas, donde se evidencia la relación entre las RRSS del Medio Ambiente (Reigota, 1990) y las emociones básicas, que el alumnado debía ir completando de manera autónoma. Esta ficha consta de tres partes: el dibujo sobre lo que es para ellos/as el Medio Ambiente; una breve explicación sobre el mismo; y marcar con una "x" la emoción que les provoca. La explicación, a pesar de ser breve, ha resultado crucial para la comprensión de algunas representaciones pictóricas, ya que nos ha ayudado a interpretarlas 
de manera correcta. Respecto al apartado enfocado a la vinculación del dibujo con el aspecto emocional, se ha tenido en cuenta la clasificación de las emociones básicas (Ekman y Oster, 1981), estableciendo a partir de la misma ocho emociones principales: alegría, tristeza, ira, culpabilidad, desprecio, asombro, serenidad y admiración. Además, se ha decidido incluir la variante "neutra" puesto que cabe la posibilidad de que el Medio Ambiente no produzca ninguna sensación en ellos. En este sentido podríamos diferenciar tres grupos: emociones positivas (alegría, asombro, serenidad y admiración), emociones negativas (tristeza, ira, culpabilidad y desprecio) y emociones neutras (ninguna).

A partir de todo ello, se podrá comprobar si las RRSS aportadas por el alumnado son favorecedoras para la implantación de la Agenda 2030 en el ámbito escolar y si las emociones vinculadas pueden llegar a generar conductas pro-activas desde la transformación ecosocial. No se trata tanto de evidenciar si se han representado los ODS, cuestión que realizaremos en investigaciones futuras, sino más bien de constatar la existencia o no de representaciones con enfoques globalizados de la dimensión natural y social por su posible coincidencia con la perspectiva holística propuesta por la Agenda 2030. A la vez que se analiza la asociación de este tipo de RRSS con las emociones señaladas por los escolares que pueden conllevar a implementar acciones transformadoras.

\section{ANÁlisis Y DISCUSIÓN DE LOS RESULTADOS: LA REPRESENTACIÓN SOCIOEMOCIONAL DEL MEDIO AMBIENTE}

En el análisis de los resultados, se ha tenido en cuenta la interpretación de las representaciones pictóricas junto a la explicación del alumnado para contrastar estos datos con las argumentaciones expuestas por Reigota (1990) sobre los tres tipos de RRSS básicas que se pueden dar sobre el Medio Ambiente: naturalista, antropocéntrica y globalizante. La asociación de las mismas con las emociones (Ekman y Oster, 1981) que ha señalado el alumnado y el enfoque idealizado o problematizado transmitido en sus dibujos quedan reflejados en la tabla 1.

TABLA 1. Relación entre emociones, representaciones sociales del Medio Ambiente y enfoques idealizados o problematizados según este estudio de casos

\begin{tabular}{|c|c|c|c|c|c|}
\hline \multirow{2}{*}{$\begin{array}{c}\text { EMOCIONES } \\
\text { (Ekman y Oster, } \\
1981)\end{array}$} & \multicolumn{3}{|c|}{$\begin{array}{l}\text { REPRESENTACIONES SOCIALES } \\
\text { (Reigota,1990) }\end{array}$} & \multicolumn{2}{|c|}{ ENFOQUES } \\
\hline & Naturalista & Antropocentrista & Globalizante & Idealizado & Problematizado \\
\hline Alegría & 28 & 6 & 13 & 34 & 13 \\
\hline Ninguna & 7 & 1 & 4 & 8 & 4 \\
\hline Tristeza & & & 8 & & 8 \\
\hline Ira & & & 1 & & 1 \\
\hline Culpabilidad & & & 14 & & 14 \\
\hline Desprecio & & & 3 & & 3 \\
\hline Asombro & 2 & & 8 & 2 & 8 \\
\hline Serenidad & 2 & & 10 & 2 & 10 \\
\hline Admiración & 2 & & 8 & 2 & 8 \\
\hline TOTALES & 41 & 7 & 69 & 48 & 69 \\
\hline $\begin{array}{l}\text { PORCENTAJE DE } \\
\text { LA MUESTRA }\end{array}$ & $35 \%$ & $6 \%$ & $59 \%$ & $41 \%$ & $59 \%$ \\
\hline $\begin{array}{c}\text { DIBUJOS } \\
\text { ANALIZADOS }\end{array}$ & \multicolumn{3}{|c|}{117} & \multicolumn{2}{|c|}{117} \\
\hline
\end{tabular}

Fuente: elaboración propia a partir de la adaptación de la clasificación de Reigota (1990) para las RRSS del Medio Ambiente y su relación con las emociones básicas de (Ekman y Oster,1981) 
Según los datos de los 117 dibujos analizados se observa, por columnas, que predominan las representaciones globalizantes y los enfoques problematizados. Sin embargo, si nos fijamos en las filas podemos distinguir que existe una relación clara entre determinadas emociones, RRSS y la idealización o problematización del medio.

La emoción de la alegría es la más destacada en RRSS naturalistas y antropocéntricas relacionadas con enfoques idealizados. A su vez, la ausencia de emoción (ninguna) es más evidente en la idealización del Medio Ambiente. Lo que conlleva a reflexionar sobre el posible desconocimiento que este alumnado pueda tener de la problemática ambiental.

Las emociones negativas (tristeza, ira, culpabilidad y desprecio) se asocian exclusivamente a RRSS globalizantes desde enfoques problematizados, cuestión que hace intuir que los niños/as que han realizado estos dibujos son conscientes de los daños que el ser humano infringe al Medio Ambiente.

Respecto a las emociones de asombro, serenidad y admiración se observa que de manera minoritaria se adscriben a representaciones naturalistas desde visiones idealizadas que bien se podrían incluir en el primer grupo descrito porque parece ser que este alumnado desconoce la problemática ambiental existente. Es interesante resaltar la relación predominante de estas emociones con las RRSS globalizantes desde enfoques problematizados porque evidencian que estos niños/as son conscientes de los acuciantes problemas socioambientales con los que vivimos hoy en día enfrentándose a ellos, desde una actitud positiva y constructiva, aportando soluciones.

Por lo tanto, a tenor de estos resultados es posible plantear tres agrupamientos (Fig. 1):

- Grupo 1: Representación social naturalista o antropocéntrica vinculada a las emociones de alegría o ninguna con un enfoque idealizado.

- Grupo 2: Representación social globalizante vinculada a las emociones negativas desde un enfoque problematizado.

- Grupo 3: Representación social globalizante relacionada con las emociones de asombro, serenidad y admiración desde un enfoque problematizado.

FIGURA 1. Distribución del número de casos por grupos resultantes de este estudio

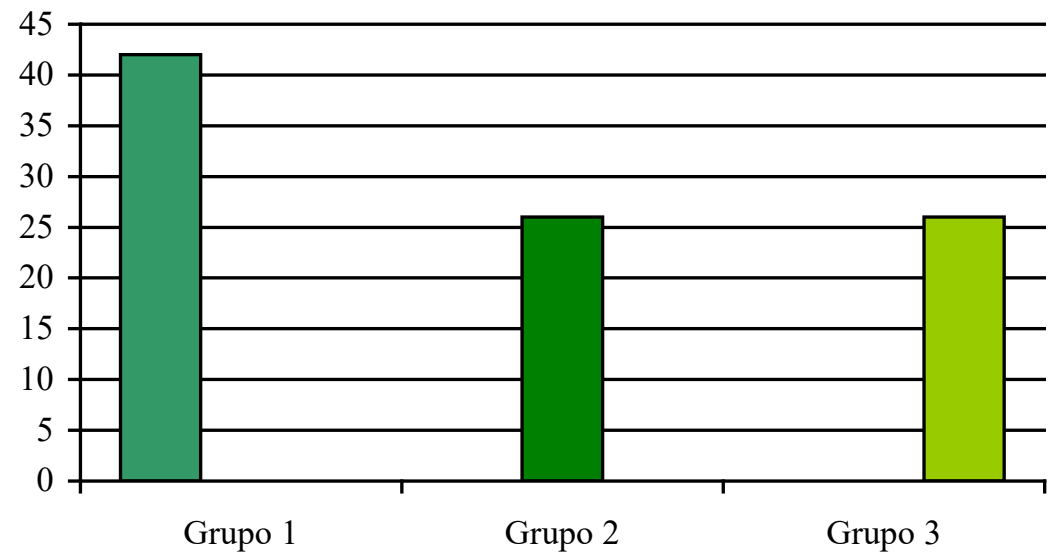

Fuente: elaboración propia
Grupo 1: Representación social naturalista/antropocéntrica con emociones de alegría o ninguna desde un enfoque idealizado

Grupo 2: Representación social globalizante con emociones negativas desde un enfoque problematizado

$\square$ Grupo 3: Representación social globalizante con emociones de asombro, serenidad y admiración desde un enfoque problematizado

Respecto a las representaciones pictóricas, en el primer grupo (Fig. 2) se introducen elementos que conforman un espacio natural que parece estar sacado de un cuento, por su carácter idílico. Encontramos elementos como árboles, plantas o animales relacionados con una representación debida a la idealización del paisaje. Esto es causado porque, posiblemente, hayan entendido el concepto de Medio Ambiente desde una perspectiva alejada de la realidad en la que 
viven, ya que no hay ninguna vinculación con el entorno. En ciertas ocasiones se compara el Medio Ambiente con la naturaleza sin el componente social, lo cual nos da indicios fiables de que se trata de una representación de tipo naturalista e idealizada.

FIGURA 2. Representación social naturalista vinculada a las emociones de alegría o ninguna con un enfoque idealizado (Grupo 1)
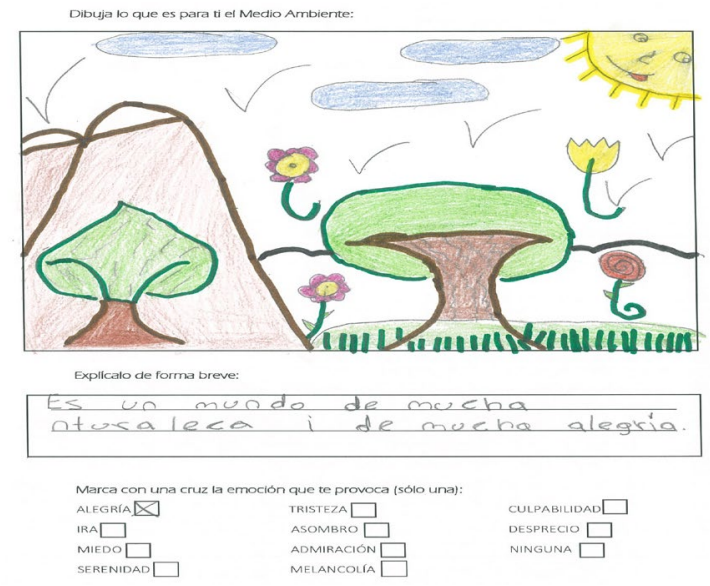

Fuente: alumno de la muestra de estudio (A-25)

Así mismo, referente al caso de la representación social antropocéntrica, encontramos ciertos dibujos en los que el alumnado comprende el Medio Ambiente desde una perspectiva antropocéntrica, en la que la idea del biocentrismo, es decir donde el hombre forma parte del entorno, no tiene cabida. Por ejemplo, en la Figura 3 el alumno hace la siguiente alusión al Medio Ambiente "Me gusta porque paso los ríos con todoterreno" (A-43). Es decir, el ser humano es capaz de transformar, modificar y organizar el medio, es el agente más poderoso de la acción ambiental.

FIGURA 3. Representación social antropocéntrica vinculada a las emociones de alegría o ninguna con un enfoque idealizado (Grupo 1)
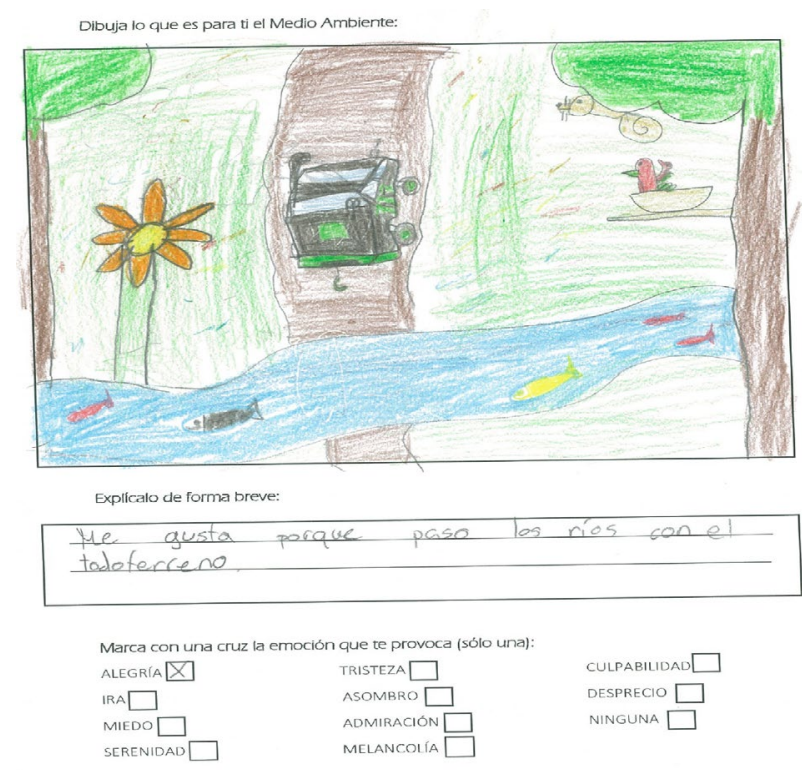

Fuente: alumno de la muestra de estudio (A-43) 
Centrándonos en el aspecto emocional, al seleccionar la casilla de alegría o ninguna reflejan que no son conocedores de las problemáticas que se generan en el entorno, y por tanto ello no incita a un desarrollo de actitudes reflexivas que lleven a la solución de estas cuestiones, ni hacia la transformación de dichos contenidos conceptuales, por lo que no se desarrolla la inteligencia ecológica manifestada por Goleman (2009). En este sentido, podemos observar que no existe ningún tipo de denuncia hacia la situación en la que se encuentra el ecosistema, o bien por desconocimiento, o bien por conformismo.

En el segundo grupo se han recogido todas las emociones negativas puesto que todas ellas van íntimamente asociadas a RRSS globalizantes de carácter problematizado (Fig. 4). En la mayoría de los dibujos aparecen temáticas de problemas medioambientales, e incluso se generan comparativas entre las buenas acciones y las actitudes que no son beneficiosas para el Medio Ambiente.

FIGURA 4. Representación social globalizante vinculada a las emociones negativas desde un enfoque problematizado (Grupo 2)

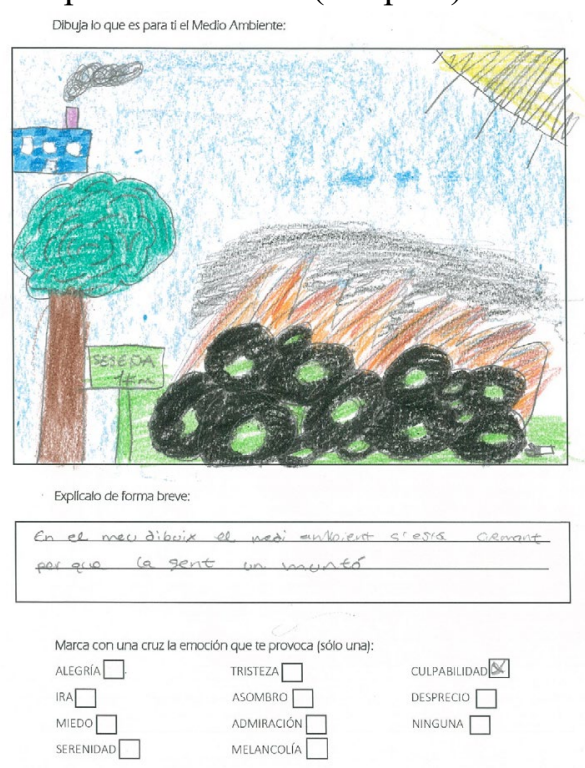

Fuente: alumno de la muestra de estudio (A-57)

Concretamente, la emoción de tristeza va asociada a problemáticas frecuentes como los incendios o la contaminación; la emoción de culpabilidad se emplea para tachar la acción antrópica como causa de la situación actual; y las emociones de ira y desprecio tienen que ver con la acción antrópica. Sin embargo, ellos mismos no se incluyen como causantes, canalizando así estas emociones a las personas que cometen esas imprudencias. Por todo ello podríamos decir que existe una vinculación evidente entre este grupo de emociones negativas y una representación social del Medio Ambiente globalizante, puesto que son capaces de extrapolar problemas que incluyen esa interdependencia entre el ser humano y el medio, además de tener un enfoque problematizado que les beneficia a la hora de detectar inconvenientes en el entorno.

En el grupo 3 las representaciones pictóricas reflejan algunas acciones y actitudes positivas que garantizan el cuidado y la prosperidad en el Medio Ambiente (Fig. 5). En contraposición a lo que ocurría en los casos anteriores, aquí el ser humano aparece como sujeto activo en la recuperación y protección del entorno, es decir, existe una relación sostenible entre el medio y el hombre, conviven en consonancia. El ser humano se integra en las relaciones ecosistémicas, que rigen el funcionamiento del planeta. 
FIGURA 5. Representación social globalizante relacionada con las emociones de asombro, serenidad y admiración desde un enfoque problematizado (Grupo 3)

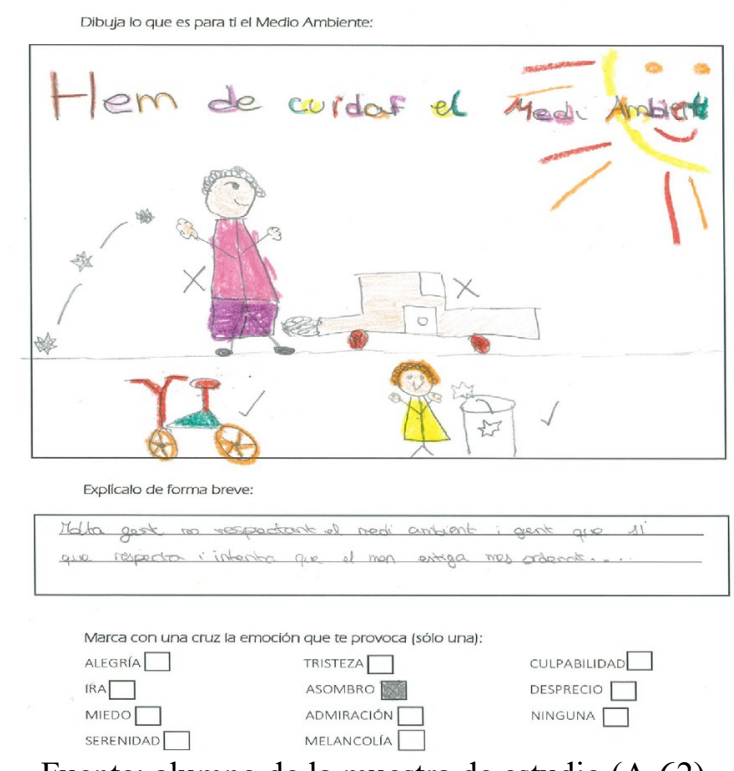

Fuente: alumno de la muestra de estudio (A-62)

Así pues, entendemos que este grupo de estudiantes ha sido capaz de desarrollar esa inteligencia emocional, de manera competente tal y como manifiesta Bisquerra (2003) porque son capaces de comprender qué consecuencias tienen sobre el Medio Ambiente las decisiones que tomamos en nuestro día a día. Por ello, podríamos decir que este grupo es capaz de mostrar una visión biocéntrica al entender que el ser humano forma parte del planeta, sin pretender una supremacía sobre el mismo, sino que ambos coexisten de manera equilibrada y sostenible. Por ello, vemos que se trata de RRSS globalizadas, en las que son capaces de pensar glocalmente, y además problematizadas, pero con una visión positiva de futuro.

Este aspecto es relevante en tanto que evidencia cómo se identifica un problema como parte de la realidad cotidiana, pero en lugar de visibilizar un componente afectivo desfavorable que se estanca en la denuncia, se incluye una visión ecosocial (González, coord., 2018) capaz de gestionar la emoción para favorecer actitudes que favorecen el cambio social (Calixto, 2010) y las vincula a prácticas sociales transformadoras. De este modo, se evidencia cómo las mismas RRSS se complementan con unas determinadas actitudes manifestadas por una reacción afectiva (Araya, 2002). Así mismo, en coherencia con las aportaciones de Amérigo, Aragonés y García (2012), se evidencia un mayor grado de inclusión del individuo en el medio natural y social, la vinculación emocional se orienta hacia la necesidad de cambio y se relaciona con la acción social (Carretero, 2010). Por tanto, merece la pena emprender una acción educativa capaz de establecer claras interdependencias entre la Educación Emocional y la Educación Ambiental, en base a desarrollar modelos emocionalmente ecológicos para favorecer no solamente la preservación del entorno (García, 2013; Soler y Conangla, 2014), sino también la ciudadanía crítica (Gil-Pérez y Vilches, 2019; Santana, Morales y Souto, 2019), desde la indisociable vinculación entre la solidaridad y la sostenibilidad (Parra, Morales y Caurín, 2020).

Las RRSS de los grupos 2 y 3 se alinean con la propuesta holística de la Agenda 2030 al representar la dimensión natural y social del Medio Ambiente, cuestión que predispone al alumnado a abordar la realidad de manera compleja. Si bien es en el grupo 3 dónde se aprecia una vinculación emocional generadora de acciones transformadoras pro-activas. Todo ello nos lleva reconsiderar la importancia de la transversalidad en Educación Ambiental (Vilches y Gil-Pérez, 2021) a través de los proyectos innovadores de transformación ecosocial. 


\section{Algunas CONSIDERACIONES PARA PROSEgUIR: ¿EXISTE UNA PAUTA EN EL APRENDIZAJE DE LA EDUCACIÓN AMBIENTAL QUE PUEDA CONTRIBUIR AL DESARROLLO DE LA AGENDA 2030?}

El análisis exploratorio de este estudio de casos con alumnado de tercer ciclo de Educación Primaria nos permite apreciar los siguientes indicios respecto a los objetivos planteados al inicio de esta investigación:

a) Respecto al análisis de las RRSS del concepto del Medio Ambiente del alumnado de tercer ciclo de Educación Primaria y su clasificación según Reigota (1990), se observa la coexistencia de RRSS globalizantes del Medio Ambiente desde enfoques problematizados con otro tipo de representaciones (naturalistas y antropocéntricas) desde visiones idealizadas.

b) En lo referente a distinguir la vinculación existente entre las distintas RRSS del Medio Ambiente y su correlación con un determinado tipo de emociones (Ekman y Oster,1981) se corrobora el surgimiento en el alumnado de emociones, tanto positivas (alegría, asombro, serenidad y admiración) como negativas (tristeza, ira, culpabilidad y desprecio) y neutras (ninguna) relacionadas con el Medio Ambiente. Verificando la relación existente entre emociones básicas (Ekman y Oster, 1981), RRSS del Medio Ambiente (Reigota, 1990) y enfoques idealizados o problematizados del medio. Desde esta perspectiva correlacional se establece la siguiente tipología:

- Grupo 1.- Representación social naturalista o antropocéntrica vinculada a las emociones de alegría o ninguna con un enfoque idealizado. La cual está condicionada por el desconocimiento de la problemática socioambiental. Éste sería el grupo más representativo.

- Grupo 2.- Representación social globalizante vinculada a las emociones negativas desde un enfoque problematizado. Como consecuencia de la percepción y concienciación de la problemática ambiental.

- Grupo 3.- Representación social globalizante relacionada con las emociones de asombro, serenidad y admiración desde un enfoque problematizado. Al vislumbrar formas sostenibles de interaccionar con el medio.

c) Respecto a la presumible incidencia de la Educación Emocional en el aprendizaje de la Educación Ambiental se evidencia una relación recíproca entre Educación Emocional y Educación Ambiental. El desconocimiento de las problemáticas ambientales suscita emociones de alegría, o ausencia de emoción, desde visiones idealizadas. Mientras que la tristeza, ira, preocupación y culpabilidad se generan desde la visión problematizada del medio. La percepción del concepto o idea de Medio Ambiente condicionará la forma en que el alumnado lo vivencia a través de las emociones que siente. Y a su vez las emociones generadas provocarán una determinada forma de comprender el entorno que repercute en su conducta prosocial y proambiental. Por lo que en definitiva la infancia vive el Medio Ambiente como lo siente y lo siente como lo vive.

A tenor de estos resultados se intuye la existencia de una serie de etapas en la interiorización de la Educación Ambiental a través de la vinculación emocional con el entorno:

- $1^{a}$ Etapa: idealización. El alumnado no es consciente de las problemáticas, vive ajeno a la realidad del entorno que le rodea y por tanto las emociones que el Medio Ambiente le evoca son positivas (alegría) o se da una ausencia de las misma (ninguna) debido al desconocimiento de la problemática socioambiental. En nuestra investigación el alumnado de esta fase se encontraría en el grupo 1.

- $2^{\text {a }}$ Etapa: problematización. El alumnado es consciente de las problemáticas ambientales, es capaz de detectar las situaciones negativas por las que atraviesa el ecosistema y las denuncia, por lo que los sentimientos que el Medio Ambiente le provocan son negativos (tristeza, ira, culpabilidad y desprecio). En esta investigación el alumnado perteneciente a esta fase se ubicaría en el grupo 2. 
- $\quad 3^{a}$ Etapa: acción ecosocial transformadora. El alumnado ya ha comprendido las situaciones negativas en las que se encuentra el planeta y toma un papel activo tratando de buscar soluciones que erradiquen esas problemáticas, por lo que su percepción del Medio Ambiente refleja emociones positivas (asombro, serenidad y admiración) ya que es capaz de ver soluciones sostenibles factibles para darle la vuelta a la grave situación medioambiental que existe en la actualidad. En nuestra investigación el alumnado perteneciente a esta fase se encontraría en el grupo 3.

Todo ello ha supuesto la verificación de la hipótesis de partida. Las prospectivas de futuro indican que para el desarrollo de la Agenda 2030 se debe fomentar la sostenibilidad y la transversalidad curricular con la finalidad de capacitar al alumnado para alcanzar la $3^{\mathrm{a}}$ etapa de interiorización de la Educación Ambiental porque además del necesario enfoque globalizado y problematizado del entorno se precisa una reflexión crítica para que la vinculación emocional lleve a la acción coherente, comprometida y responsable con el Medio Ambiente que abarque la dimensión ecológica y social.

A modo de conclusión final es preciso alinear agendas institucionales, escolares y personales en relación con los propósitos de transformación del mundo que nos ofrecen los ODS. Pero el reto, tal y como demuestra este estudio, es saber en qué etapa está nuestro alumnado para comprender, alcanzar y superar la propuesta de la Agenda 2030, la cual debe ser entendida como una hoja de ruta siempre mejorable.

\section{Referencias}

Abric, J. C. (2001). Prácticas sociales y representaciones. México: Coyoacán.

Amérigo, M., Aragonés, J. I. y García, J. A. (2012). Explorando las dimensiones de la preocupación ambiental. Una propuesta integradora. Psyecology, 3(3), 299-311. DOI: 10.1174/217119712802845705

Amérigo, M., García, J. A. y Sánchez, T. (2013). Actitudes y comportamiento hacia el medio ambiente natural. Salud medioambiental y bienestar emocional. Universitas Psychologica, 12(3), 845- 856. DOI: 10.11144/javeriana.upsy12-3.acma

Araya, S. (2002). Las representaciones sociales: ejes teóricos para su discusión. Cuaderno de Ciencias Sociales 127. Facultad Latinoamericana de Ciencias Sociales (FLACSO). Recuperado de https://goo.gl/yCqq7B

Bautista-Cerro, M. J., Murga-Menoyo, M. A. y Novo, M. (2019). La Educación Ambiental (página en construcción, disculpen las molestias). Revista de Educación Ambiental y Sostenibilidad 1(1), 1103. DOI: 10.25267/Rev_educ_ambient_sostenibilidad.2019.v1.i1.1103

Barraza, L. (1999). Children's drawing about the environment. Environmental Education Research. Bath. 5(1), 49-67.

Bisquerra, R. (2003). Educación emocional y competencias para la vida. Revista de Investigación Educativa, 21(1), 7-43.

Bisquerra, R. (2006). Orientación psicopedagógica y educación emocional. Estudios sobre Educación, 11, 9-25.

Calixto, R. (2010). Medio ambiente y educación ambiental: representaciones sociales de los profesores en formación. Magis: Revista Internacional de Investigación en Educación, 2(4), 401-414.

Carretero, A. (2010). Para una tipología de las «representaciones sociales»: una lectura de sus implicaciones epistemológicas. EMPIRIA: Revista de Metodología de Ciencias Sociales, 20, 87-108. DOI: 10.5944/empiria.20.2010.2041

Durkheim, E. (1898). Représentations individuelles et représentations collectives. Revue de Métaphysique et de Morale, 6(3), 273-302. 
Ekman, P. y Oster, H. (1981). Expresiones faciales de la emoción. Estudios de Psicología, 2(7), 115-144. DOI: $10.1080 / 02109395.1981 .10821273$

Frijda, N. H. (1988). The laws of emotion. American Psychologist, 43, 349-358. DOI: 10.1037/0003-066x.43.5.349

García, A. L. (2013). El proceso de desarrollo de los Itinerarios Geográficos. Bienvenidos, 2, 3-9.

García, D., Morote, A. y Souto, X. M. (2019). Las representaciones del saber académico: aportaciones desde la geografía escolar. Arxius de Ciències Socials, 41, 11-28.

Gil-Pérez, D. y Vilches, A. (2019). La comprensión e impulso de la Sostenibilidad: un requisito imprescindible para una acción educativa y ciudadana eficaz. Revista de Educación Ambiental y Sostenibilidad 1(2). DOI: 10.25267Rev_educ_ambient_sostenibilidad.2019.v1.i2.2101

Goleman, D. (2009). Inteligencia Ecológica. Kairós.

González, L. (coord.) (2018). Educar para la transformación ecosocial. Orientaciones para la incorporación de la dimensión ecosocial al currículo. Madrid: FUHEM.

Hartig, T., Kaiser, F. G. y Bowler, P. A. (2001). Psychological restoration in nature as a positive motivation for ecological behavior. Environment \& Behavior, 33(4), 590-607. DOI: $10.1177 / 00139160121973142$

Jodelet, D. (1986). La representación social: fenómeno, concepto y teoría. En S. Moscovici (Ed.), Psicología Social. II (pp. 470-494). Paidós.

Jodelet, D. (1994). Les représentations sociales. PUF.

Lindenberg, S. y Steg, L. (2007). Normative, gain and hedonic goal frames guiding environmental behavior. Journal of Social Issues, 63(1), 117-137. DOI: 10.1111/j.1540-4560.2007.00499.x

Milton, K. (2002). Loving nature. Towards and ecology of emotion. Routledge.

Morales, A.J., Caurín, C., Sendra, C. y Parra, D. (2014). Aprendiendo a plantear problemas en el medio. Análisis de una experiencia con estudiantes del Máster de Investigación en Didácticas específicas. Didáctica de las Ciencias Experimentales y Sociales, 28, 65-81. DOI: $10.7203 /$ dces. 28.3848

Morales, A.J., Santana, D. y Sánchez, L. (2017). Identidades territoriales y educación ambiental. Del paisaje emocional al paisaje cultural. Íber, Didáctica de las Ciencias Sociales, Geografía e Historia, 89, 12-17.

Moscovici, S. (1961). La psychanalyse, son image et son public: étude sur la représentation sociale de la psychanalyse. París: Presses Universitaires de France.

Moscovici, S. (1979). El psicoanálisis, su imagen y su público. Buenos Aires: Huemul.

Naciones Unidas (2015). Transformar nuestro mundo: la Agenda 2030 para el desarrollo sostenible. Recuperado https://unctad.org/meetings/es/SessionalDocuments/ares70d1_es.pdf.

Nundy, S. (1999). The fieldwork effect: the role and impact of fieldwork in the upper primary school'. International Research in Geographical and Environmental Education, 8(2), 190-8. DOI: $10.1080 / 10382049908667608$

Parra, A., Morales, A.J. y Caurín, C. (2020). La solidaridad y la sostenibilidad a través de los docentes con formación en mediación: ¿Una realidad indisociable? Didáctica de las Ciencias Experimentales y Sociales, 38, 61-80. DOI: 10.7203/DCES.38.13828.

Puig, E., Geli, A. M. y Besalú, X. (2018). Percepción y proyección de los problemas sociales y ambientales. Didáctica de las ciencias experimentales y sociales, 35, 91-112. DOI: 10.7203/DCES.35.12347

Reigota, M. (1990). Las representaciones sociales del medio ambiente y las prácticas pedagógicas cotidianas de los docentes de ciencia en Sao Paulo, Brasil. Tesis Doctoral. Universidad Católica de Lovaina.

Reigota, M. (2010). Las representaciones sociales como práctica pedagógica cotidiana de la educación ambiental. Investigación y Educación Ambiental, 71, 71-79. 
Sánchez, L. (2016). Las representaciones sociales del concepto de medio ambiente y su incidencia emocional en el tercer ciclo de Educación Primaria. Estudio de casos. Trabajo Final de Máster. Universitat de València. Inédito.

Santana, D., Morales, A.J. y Henarejos, I. (2021). Educación Ambiental y proyectos educativos. Iniciativas escolares de transformación ecosocial. Íber, Didáctica de las Ciencias Sociales, Geografia e Historia, 102, 20-26.

Santana, D., Morales, A. J. y Souto, X. M. (2019). ¿Cómo favorecer la ciudadanía critica con la ayuda de la educación ambiental? En M. Ferreras-Listán, O. Moreno-Fernández y M. a . PuigGutiérrez (coords.), Innovación e investigación en la formación inicial del profesorado de Infantil y Primaria desde las didácticas de las Ciencias Experimentales y Sociales (pp. 77100). Barcelona: Octaedro.

Scott, W. (2010). La investigación y la educación ambiental: La necesidad de apuestas

multidimensionales. En M. Torres, A. M. Vergara y J. Cárdenas (Coords.), Investigación y Educación Ambiental. Apuestas investigativas pertinentes a los campos de reflexión e intervención en educación ambiental, cap. 1 (pp. 23-37). Universidad Distrital Francisco José de Caldas.

Seresinhe, C. I., Preis, T. y Moat, H. S. (2015). Quantifying the impact of scenic environments on health. Scientific reports, 5. DOI: 10.1038/srep16899.

Soler, J. y Conangla, M. (2013). Ecología Emocional. Amat Editorial.

Souto, X. M. (2011). Identidades espaciales y territorios políticos. Íber, Didáctica de las Ciencias Sociales, Geografia e Historia, 69, 55-63.

Souto, X. M. y García, D. (2017). La geografía escolar ante el espejo de su representación social. Didáctica Geográfica, (17), 177-201.

Souto, X.M. y García, D. (2019). Conocer las rutinas para innovar en la geografía escolar. Revista de Geografía Norte Grande, 74, 207-228. DOI: 10.4067/S071834022019000300207

Stake, R.E. (1999). Investigación con estudio de casos. Madrid: Ediciones Morata S.L.

Tonucci (1993). Hacia una propuesta de educación ambiental coherente. Aula de Innovación Educativa, 12, 82-85.

Unesco (1977). Conferencia Intergubernamental sobre Educación Ambiental. Organizado por la Unesco en cooperación con la PNUMA. Tbilisi. Recuperado de http://unesdoc.unesco.org/images/0003/000327/032763sb.pdf (4-1.2018)

Vilches, A. y Gil-Pérez, D. (2021). Contribución de la educación a la transición a la sostenibilidad. Íber, Didáctica de las Ciencias Sociales, Geografia e Historia, 102, 8-14.

Villarroel, G. (2007). Las representaciones sociales: una nueva relación entre el individuo y la sociedad. Fermentum. Revista Venezolana de Sociología y Antropología, 17(49), 434-454.

Yin, R. K. (1981). The Case Study Crisis: Some Answers. Administrative Science Quarterly, 26(1), 58-65. DOI: $10.2307 / 2392599$

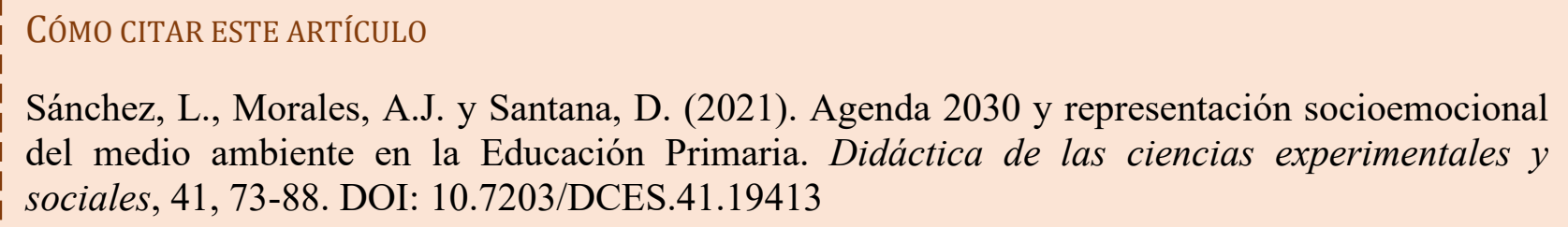


